\title{
MSC Therapeutics in Chronic Inflammation
}

\author{
Alex Sargent ${ }^{1} \cdot$ Robert H. Miller ${ }^{2}$
}

Published online: 29 March 2016

(C) Springer International Publishing AG 2016

\begin{abstract}
The utilization of mesenchymal stem cells (also known as mesenchymal stromal cells (MSCs)) as a cellbased therapy for diseases that have ongoing inflammatory damage has become increasingly available. Our understanding of the cell biology of MSCs is still incomplete. However, as a result of increasing numbers of preclinical and clinical studies, general themes are emerging. The capacity of MSCs to reduce disease burden is largely associated with their ability to modulate the activity of the host immune responses rather than to contribute directly to tissue regeneration. As a result, they have significant potential in the treatment of chronic inflammatory disease regardless of the affected tissue. For example, MSC-based therapies have been developed in the context of diseases as diverse as rheumatoid arthritis and multiple sclerosis. Here, we discuss some of the principles that link these conditions and the aspects of MSC biology that contribute to their use as a therapy for chronic inflammatory conditions.
\end{abstract}

Keywords Mesenchymal stem cells · Immune suppression · Multiple sclerosis · EAE

This article is part of the Topical Collection on Stem Cells: Policies from the Bench to the Clinic

Robert H. Miller

Rhm3@gwu.edu

Alex Sargent

Ags60@case.edu

1 Department of Neurosciences, Case Western Reserve University, School of Medicine, 2210 Circle Drive, Cleveland, OH 44106, USA

2 Department of Anatomy and Regenerative Medicine, George Washington University, School of Medicine and Health Sciences, 2300 Eye Street NW, Ross Hall 709G, Washington DC 20037, USA

\section{Introduction}

\section{Properties of Mesenchymal Stem Cells}

The early identification and characterization of mesenchymal stem cells depended on the development of differential adhesion and survival conditions for cultured bone marrow cells. With extended culture intervals of bone marrow-derived cells under select conditions, colonies of cells developed that appeared to contain multipotent, self-renewing cells with the capacity to differentiate into the different mesenchymal cell derivatives: fat, cartilage, and muscle depending on the culture conditions [1]. Whether these cells are a significant source of mesenchymal tissue replacement in the adult is unclear but seems unlikely. Mesenchymal stem cells are not restricted to bone marrow and may be equivalent to pericytes [2,3], which line the vasculature of most tissues. Indeed, mesenchymal stem cells (MSCs) for therapeutic purposes have been isolated from adipose and other highly vascularized tissue [2]. Several roles have been proposed for MSCs in bone marrow including that they contribute to the hematopoietic stem cell (HSC) niche by providing survival and mobilization cues to HSCs in response to environmental cues. A major confounding factor in developing a clear understanding of normal MSC biology has been the lack of a single definitive marker to isolate and identify these cells in vivo, although combinations of surface markers have been defined. A consensus statement from the International Society for cellular therapy established the following minimal criteria as defining mesenchymal stromal cells: (1) a cell population adherent to standard tissue culture plastic; (2) expression of the cell surface epitopes CD105, CD90, and CD73; (3) lack of expression of the cell surface epitopes CD45, CD11b, CD34, and Cd14; and (4) ability to differentiate into osteoblasts, adipocytes, and chondrocytes under suitable culture conditions [4]. The lack 
of definitive markers for MSCs has not hindered the application of these cells to the clinic. Because of the relative ease of their availability, the refinement of growth conditions, and the ability in chronic diseases to use autologous rather than allogeneic cells for infusion, MSCs have moved rapidly from the laboratory to the clinic, and there are a large number of ongoing MSC clinical trials in a wide range of different disease conditions $[5,6]$.

The biology of MSCs is complicated. Perhaps more than most other cell types, they have the potential to express an extremely broad array of growth factors and cytokines [5]. These include both anti-inflammatory as well as proinflammatory cytokines and a spectrum of chemokines and growth factors such as fibroblast growth factors (FGFs), hepatocyte growth factor (HGF), and various neurotrophins such as brain-derived neurotrophic factor (BDNF) and nerve growth factor (NGF). The precise spectrum of signaling molecules expressed by MSCs appears to depend on their environment, and as a result, MSCs are able to respond to an extensive array of different environmental cues. Several lines of evidence suggest that MSCs are relatively immune privileged. They express relatively low levels of major histocompatibility antigens (MHCs) [7] and survive longer when transplanted to an allogeneic host than most other cell types [8]. The capacity of MSCs to detect changes in their environment is mediated through an extensive array of receptors for signaling molecules.

Although the biology of endogenous MSCs is still not clearly understood, an emerging concept is that these cells contribute to the stem cell niche in multiple tissues and orchestrate responses to tissue injury. Many of the insights into MSC function have come from analyses of their ability to modulate disease progression in a variety of conditions $[6,9$, 10]. Here, we discuss two chronic conditions: rheumatoid arthritis (RA) and multiple sclerosis. While each of these diseases affects different tissue targets, they share a number of important characteristics. Both conditions are characterized by chronic inflammation and local tissue destruction. In both conditions, the initial trigger for the disease is not clearly defined, although considerable evidence supports a central role for $\mathrm{T}$ cells in driving disease pathology. Finally, in both diseases, experimental data suggest that treatment with MSCs may contribute to augmentation of suppression of immunological attack as well as contribute to tissue repair.

\section{Application of MSCs to the Treatment of Rheumatoid Arthritis}

RA is a chronic inflammatory disease that affects the joints [11]. This inflammatory response results in tissue damage and secondary progressive inflammation that result in damage or loss to different tissues including cartilage and bone [11]. The stimulus that initiates the immune attack in RA is not well defined [12] and may be antigenically restricted; however, there is evidence that as the disease progresses, the spectrum of immunogenic epitopes spreads or increases and the exposure to additional epitopes contributes to ongoing disease. While multiple cell types including neutrophils, macrophages, and fibroblast-like synoviocytes (FLSs) contribute to tissue damage, several lines of evidence suggest that $\mathrm{T}$ cells are the major effectors of disease in RA [12]. Large numbers of $\mathrm{T}$ cells, of which the majority have a Th1 phenotype [13], are found in inflamed joints and are recruited through the local expression of an array of cytokines and chemokines. The infiltrating $\mathrm{T}$ cells are thought to stimulate other immune cells including mononuclear cells, macrophages, and FLS that contribute to further tissue damage.

Multiple pro-inflammatory cytokines are elevated in RA [14] including tumor necrosis factor alpha (TNF $\alpha)$ [15], which plays a major role in disease pathogenesis. Indeed, anti-TNF $\alpha$ treatments have demonstrated significant benefit in reducing inflammation in RA patients [14], and neutralizing $\mathrm{TNF} \alpha$ with humanized antibodies reduces disease progression $[16,17]$.

$\mathrm{RA}$ represents an interesting target for the application of MSCs.

MSCs are highly immunomodulatory and can inhibit the proliferation and activation of a variety of immune cells including T cells [18], which are thought to be major drivers of tissue damage and disease pathology in RA [12]. Several studies have shown that MSCs can modulate $\mathrm{T}$ cells in animal models of RA and can improve clinical recovery [19, 20•, 21]. Systemically transplanting MSCs into rodents with the collagen-induced arthritic (CIA) model of RA leads to a decrease in the number of circulating Th1-polarized T cells and an increase in the number of Th2-polarized regulatory $\mathrm{T}$ cells $[19,20 \bullet, 21]$. This modulation of T cells by MSCs in CIA mice is paralleled by improvements in tissue integrity and clinical recovery $[20 \bullet, 21]$. How MSCs might influence other types of immune cells such as macrophages or FLS in CIA is unclear, but these studies demonstrate that MSCs can improve recovery in animal models of RA by apparently modulating $\mathrm{T}$ cells and the immune response.

Not only are MSCs immunoregulatory and thus able to influence the pathogenic process but they may also be capable of differentiating into cells of the tissues damaged by the disease (such as bone and cartilage) and could thereby directly contribute to tissue regeneration. As a result, several studies have attempted to deliver MSCs directly into the damaged area with the hope that they will directly facilitate repair. It seems likely that in order for such an approach to be effective, the transplanted MSCs have to be retained in the location of the inflamed joint. One approach that has been effective in animal models is to use a scaffold composed of biodegradable nanofibers to localize MSCs [22, 23•]. Such an approach 
results in greater retention of the cells in the target joint and reduction in the inflammatory response [23•], but evidence for a direct contribution of the transplanted cells or their progeny in tissue repair is still lacking.

Future studies focused on improving the efficacy of MSC therapies for RA will need to look at enhancing local delivery and retention of MSCs in inflamed joints, where they can affect the local environment and perhaps better contribute, directly or indirectly, to tissue repair. Further characterizing the signaling pathways that mediate MSC-induced recovery in RA can also help us better understand how MSCs alter inflammation and the immune response in RA, which may lead to a better understanding of the immunopathology of RA and how to treat it. Another attractive therapeutic avenue that remains unexplored is combining effective systemic anticytokine treatments such as anti-TNF $\alpha$ with local delivery and retention of MSCs in inflamed joints where they can augment tissue repair.

\section{Application of MSCs to the Treatment of Multiple Sclerosis}

Multiple sclerosis (MS) is a chronic autoimmune disease of the central nervous system that is often diagnosed in young adulthood [24]. Characteristically, MS initially presents with transient functional deficits that affect a specific modality. The functional deficit correlates with the location of a discrete lesion within the CNS. For example, lesions in the optic nerve or optic tract result in loss of visual acuity, while damage to the spinal cord white matter results in compromised limb function. Early disease is often associated with relapses followed by periods of remission (relapsing-remitting MS). This phase of the disease is characterized by active inflammation in CNS white matter and the formation of multiple sclerotic plaques or lesions [24]. The CNS lesions in MS are characterized by a loss of oligodendrocytes and their myelin sheaths [25]. Since myelination is required for the rapid and efficient conduction of information along axons, its loss results in impaired conduction in the affected axons. Much of our mechanistic understanding of the pathobiology of MS comes from animal models. The most commonly used animal model of MS is experimental allergic encephalitis (EAE) [26]. In appropriate strains of mice, immunization with specific peptides of myelin proteins results in a CNS-directed inflammatory response. For example, immunization of C57/B16 mice with a peptide of myelin oligodendrocyte glycoprotein (MOG peptide containing amino acids $35-55$ ) results in an inflammatory demyelinating disease with a chronic course. The demyelination in this model is a result of infiltration of $\mathrm{T}$ cells targeted against myelin and oligodendrocytes [26]. Like RA, the initial trigger of disease in MS is not well characterized, but as disease progresses, at least in EAE, there appears to be epitope spreading such that the profile of antigens recognized by pathogenic T cells expands. The spinal cord is severely affected in these animals, and they develop functional motor deficits that can be easily scored.

Classic studies by Uccelli and colleagues [27-29] demonstrated that infusion of MSCs via intravenous delivery into this animal model prior to disease onset blocked disease development and MSC infusion at the peak of disease significantly reduced disease severity. These data have been replicated by several other laboratories and provide compelling evidence that MSC transplantation can effectively modulate disease progression in EAE. However, the underlying mechanisms that contribute to the MSC-mediated reduction in disease burden are not clearly defined. One general finding is that MSC treatment modulates the immune response [27, 30] and reduces the expression of pro-inflammatory cytokines, biasing the immune response from a predominantly pro-inflammatory $\mathrm{TH} 1$ profile to a predominantly anti-inflammatory $\mathrm{TH} 2$ profile (Fig. 1) [31•, 32].

The efficacy of MSCs to modulate disease progression in EAE appears to be a general phenomenon that crosses species. For example, when human bone marrow-derived MSCs are injected intravenously into mice with EAE, there is rapid functional recovery that is sustained for an extended period $[31 \bullet$, 32, 33]. Cell tracking studies suggest that at least some of the MSCs track to the CNS where they are retained for at least a few days [32]. Whether this retention is a consequence of the low level of expression of MHC epitopes on the surface of MSCs [34] or their specific localization to the CNS behind an effective blood brain barrier is unclear. More importantly, it is unknown whether cells delivered by an intravenous pathway have to enter the CNS in order to effectively reduce disease burden in EAE. Given the relatively low number of cells found in the CNS, they must clearly have a major role in modulation of the peripheral immune response [27]. Many of the effects of MSCs in alleviating disease progression in EAE appear to be mediated by secreted factors rather than by cell-cell contact. Conditioned medium (CM) collected from human bone marrow MSCs is sufficient to reduce disease burden when injected intravenously in MOG 35-55 EAE [31•]. The route of delivery appears to be important, as intraperitoneal delivery was considerably less effective than intraventricular injection. As with cell delivery, the effects of treatment with MSC-CM are relatively long lasting suggesting that there is a physiological change in the host as a consequence of exposure to MSC-CM. The long-lasting effects raise questions in the treatment of EAE of what are the target tissues for MSC-derived factors and the nature of those factors. As discussed above, MSCs have profound effects on the immune system, and not surprisingly, MSC-CM has similar effects [31•]. Recent studies suggest, however, that MSCs and their secreted products may also directly affect neural tissue (Fig. 1) $[31 \cdot, 35,36]$. Analysis of the generation of different neural 


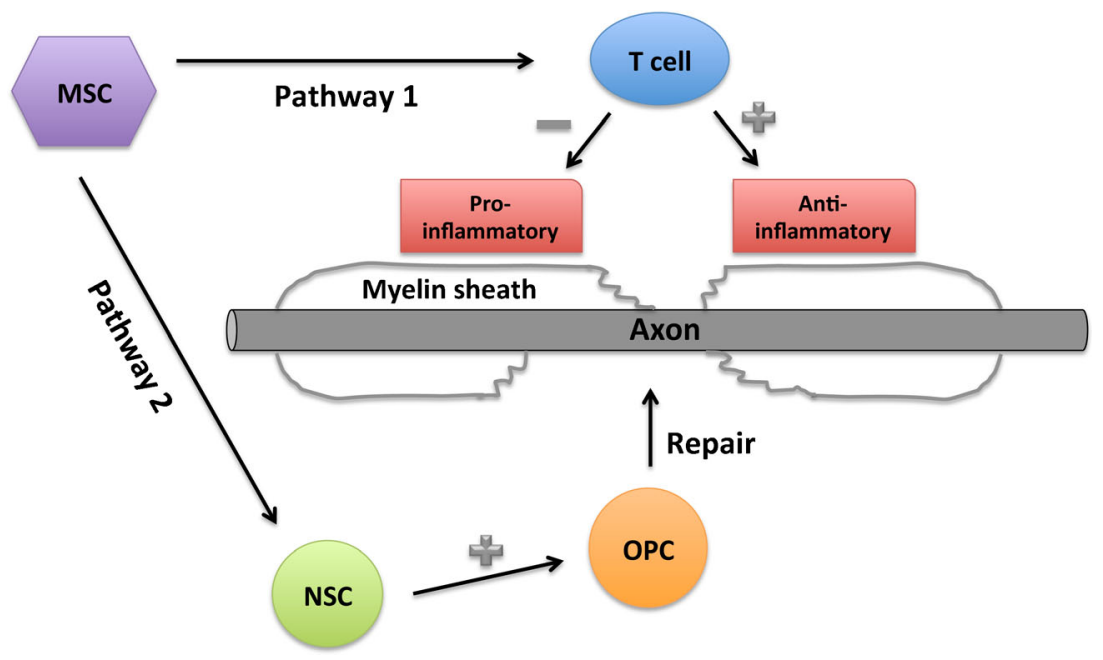

Fig. 1 The chronic inflammatory disease multiple sclerosis is characterized by local loss of oligodendrocytes and their myelin sheaths. This loss exposes axons that are then vulnerable to further damage resulting in functional loss. Infiltration of pro-inflammatory $\mathrm{T}$ cells that target myelin antigens is a major cause of demyelination. Treatment with mesenchymal stem cells (MSCs) may promote functional recovery in MS and animal models by two independent pathways. In pathway 1 , MSCs modulate the immune response in part by affecting $\mathrm{T}$ cell function. In the absence of MSCs, the majority of T cells are proinflammatory and drive myelin loss; in the presence of MSCs, T cells are induced to a more anti-inflammatory phenotype thereby reducing immunological destruction. In pathway 2, MSCs may influence neural stem cells to generate increased numbers of oligodendrocyte precursor cells that, in turn, differentiate into oligodendrocytes and repair damaged myelin. NSC neural stem cells, OPCs oligodendrocyte precursor cells lineages from neural stem cells treated with MSC-CM in vitro shows that treatment with MSC-CM results in an increased proportion of oligodendrocyte lineage cells and a reduction in astrocytes [31•]. Consistent with these data, MSC treatment results in reduced expression of RNA for glial fibrillary acidic protein (GFAP), a characteristic of astrocytes. One potential component of MSC-CM that appears to be important for the therapeutic efficacy of MSCs in EAE is HGF [31•], and overexpression of $\mathrm{HGF}$ in the CNS results in animals that are refractory to the induction of EAE [37].

The concept that MSCs may have a direct effect on neural cells and may promote the generation of oligodendrocytes and recovery of demyelinating lesions even in non-inflammatory models of demyelination $[32,38]$ raises the possibility that MSCs may be effective in treating chronic progressive multiple sclerosis. Current therapies for MS are highly effective at modulating the inflammatory attack that characterizes relapsing-remitting MS but have little or no effect on progressive MS where the level of CNS inflammation may be significantly lower but oligodendrocyte loss and demyelination are persistent. Thus, therapies like MSC infusion that could potentially target both a reduction in inflammation and an enhancement in myelin repair are an attractive candidate for the treatment of chronic progressive MS.

The application of MSC therapy to the clinical treatment of MS has moved rapidly in recent years, and there are multiple ongoing clinical trials [39-41]. Although the outcomes of the trials are not yet complete, trends are emerging. In general, MSC therapy appears safe, and there have been few reports of adverse effects [40, 41]. Although some trials have reported modest improvement, compared to the outcomes seen in animal models however, the efficacy of the MSC treatment has been somewhat disappointing [40]. A challenge has been to clearly define any meaningful improvement in patients receiving MSCs without long-term follow-up. In relapsing-remitting patients, a reduction in the number of relapses may indicate benefit, but this measure is complicated by co-treatment with other effective anti-inflammatory therapies, while in chronic MS, there are few easily measurable therapeutic outcomes other than reduction in brain atrophy or improvement in axonal transmission. In a recent proof-of-principle trial in optic neuritis, some benefit in visual acuity was detected following treatment with autologous MSCs; however, the benefit was transient [39]. Several reasons may account for the apparent lack of efficacy in the treatment of MS with MSCs. These include the source of cells, conditions of expansion, route of delivery, dosage, and characteristics of the host. The lack of a reliable biomarker that correlates with reparative capacity of distinct populations of MSCs in MS remains a major impediment to successful trials. Indeed, it is unclear whether the animal models currently used to screen MSC function such as MOG 35-55 EAE are selecting for the most effective cell populations. As the number of clinical trials testing the efficacy of MSCs in treating MS continues to increase, new insights from such work will help inform the design of new culture conditions and animal models that, in turn, will allow for more effective design of clinical trials for the treatment of chronic MS with MSCs. 


\section{Complications of MSC Therapies}

As discussed above, while MSCs have tremendous potential as a cellular therapy for chronic inflammatory diseases, the clinical trials are still at a very early stage, and it seems likely that efficacy will improve with refinement of route of delivery, dosage, and a greater understanding of the biology of MSCs. The data in a range of animal models is very encouraging, but the transition to the treatment of human disease is not simple. A variety of factors complicate patient treatment with MSCs that are simply not accounted for in animal models. These include issues of scale. The relatively small lesions generated in the CNS of mice may be vastly easier to repair than the large lesions in human white or gray matter. The response of human immune cells to MSC-derived signal may be different from that in animal models, and likewise, the response of human neural cells may be different from that in animal cells. In addition to such methodological considerations, there are other issues associated with MSC cell therapies. Given the modulation of the immune system, the changes in T cell responses, particularly alterations in the responses of circulating Treg cells, may need to be monitored. The safety and potential side effects of MSC infusion in humans also need to be better understood. For instance, whether MSC treatment suppresses the immune system to a degree that increases the risk of infection (e.g., pneumonia) needs to be assessed, as does the potential for increased incidence of tumor formation. Thus far, the data from ongoing clinical trials suggests that these potential complications are relatively minimal. If however the treatment of chronic conditions requires multiple infusions of MSCs over a protracted period of time, then the risk of adverse events clearly increases.

\section{Conclusions}

While illnesses characterized by chronic inflammation have proven difficult to control, the combined immunomodulatory and regenerative capabilities of MSCs make them a promising new candidate for the long-term treatment of inflammatory diseases including RA and MS. By better defining the molecular mechanisms by which MSCs modulate inflammation and the immune system and by better understanding the capacity of MSCs to drive endogenous tissue repair, we can better realize the potential of MSCs as novel cellular therapy for the treatment of chronic inflammation across a wide spectrum of disease.

Acknowledgments Drs. Caplan and Bonfield wish to thank Dr. Amy DiMarino for their kind assistance in the reviewing of this article.

\section{Compliance with Ethics Guidelines}

Conflict of Interest Alex Sargent and Robert H. Miller declare that they have no conflict of interest.
Human and Animal Rights and Informed Consent This article does not contain any studies with human or animal subjects performed by any of the authors.

\section{References}

Papers of particular interest, published recently, have been highlighted as:

- Of importance

1. Pittenger MF, Mackay AM, Beck SC, Jaiswal RK, Douglas R, Mosca JD, et al. Multilineage potential of adult human mesenchymal stem cells. Science. 1999;284(5411):143-7.

2. Crisan M, Yap S, Casteilla L, Chen CW, Corselli M, Park TS, et al. A perivascular origin for mesenchymal stem cells in multiple human organs. Cell Stem Cell. 2008;3(3):301-13. doi:10.1016/j.stem. 2008.07.003.

3. Caplan AI. All MSCs are pericytes? Cell Stem Cell. 2008;3(3): 229-30. doi:10.1016/j.stem.2008.08.008.

4. Dominici M, Le Blanc K, Mueller I, Slaper-Cortenbach I, Marini F, Krause D, et al. Minimal criteria for defining multipotent mesenchymal stromal cells. The International Society for Cellular Therapy position statement. Cytotherapy. 2006;8(4):315-7. doi: 10.1080/14653240600855905.

5. Caplan AI, Correa D. The MSC: an injury drugstore. Cell Stem Cell. 2011;9(1):11-5. doi:10.1016/j.stem.2011.06.008.

6. Dimarino AM, Caplan AI, Bonfield TL. Mesenchymal stem cells in tissue repair. Front Immunol. 2013;4:201. doi:10.3389/fimmu. 2013.00201.

7. Patel SA, Sherman L, Munoz J, Rameshwar P. Immunological properties of mesenchymal stem cells and clinical implications. Arch Immunol Ther Exp (Warsz). 2008;56(1):1-8. doi:10.1007/ s00005-008-0001-x.

8. Le Blanc K, Frassoni F, Ball L, Locatelli F, Roelofs H, Lewis I, et al. Mesenchymal stem cells for treatment of steroid-resistant, severe, acute graft-versus-host disease: a phase II study. Lancet. 2008;371(9624):1579-86. doi:10.1016/S0140-6736(08)60690-X.

9. Prockop DJ. Marrow stromal cells as stem cells for nonhematopoietic tissues. Science. 1997;276(5309):71-4.

10. Caplan AI, Bruder SP. Mesenchymal stem cells: building blocks for molecular medicine in the 21 st century. Trends Mol Med. 2001;7(6):259-64.

11. McInnes IB, Schett G. The pathogenesis of rheumatoid arthritis. N Engl J Med. 2011;365(23):2205-19. doi:10.1056/ NEJMra100496510.7748/phc2011.11.21.9.29.c8797.

12. Panayi GS, Corrigall VM, Pitzalis C. Pathogenesis of rheumatoid arthritis. The role of T cells and other beasts. Rheum Dis Clin North Am. 2001;27(2):317-34.

13. Pitzalis C, Kingsley G, Murphy J, Panayi G. Abnormal distribution of the helper-inducer and suppressor-inducer T-lymphocyte subsets in the rheumatoid joint. Clin Immunol Immunopathol. 1987;45(2): 252-8.

14. Feldmann M, Maini SR. Role of cytokines in rheumatoid arthritis: an education in pathophysiology and therapeutics. Immunol Rev. 2008;223:7-19. doi:10.1111/j.1600-065X.2008.00626.x.

15. Elliot MJ, Maini RN, Feldmann M, Long-Fox A, Charles P, Katasikis $\mathrm{P}$, et al. Treatment of rheumatoid arthritis with chimeric monoclonal antibodies to tumor necrosis factor alpha. Arthritis Rheum. 2008;58(2 Suppl):S92-101. doi:10.1002/art.23362. 
16. Kavanaugh AF. Anti-tumor necrosis factor-alpha monoclonal antibody therapy for rheumatoid arthritis. Rheum Dis Clin North Am. 1998;24(3):593-614.

17. Moreland LW. Inhibitors of tumor necrosis factor for rheumatoid arthritis. J Rheumatol Suppl. 1999;57:7-15.

18. Duffy MM, Ritter T, Ceredig R, Griffin MD. Mesenchymal stem cell effects on T-cell effector pathways. Stem Cell Res Ther. 2011;2(4):34. doi:10.1186/scrt75.

19. Gonzalez MA, Gonzalez-Rey E, Rico L, Buscher D, Delgado M. Treatment of experimental arthritis by inducing immune tolerance with human adipose-derived mesenchymal stem cells. Arthritis Rheum. 2009;60(4):1006-19. doi:10.1002/art.24405.

20. Lopez-Santalla M, Mancheno-Corvo P, Menta R, Lopez-Belmonte J, DelaRosa O, Bueren JA, et al. Human adipose-derived mesenchymal stem cells modulate experimental autoimmune arthritis by modifying early adaptive T cell responses. Stem Cells. 2015;33(12): 3493-503. doi:10.1002/stem.2113. This study showed that MSCs improve recovery in the rodent CIA model of RA by reducing the number of pathogenic Th1-cells and increasing the number of regulatory T-cells.

21. Gu J, Gu W, Lin C, Gu H, Wu W, Yin J, et al. Human umbilical cord mesenchymal stem cells improve the immune-associated inflammatory and prothrombotic state in collagen type-induced arthritic rats. Mol Med Rep. 2015;12(5):7463-70. doi:10.3892/mmr.2015. 4394.

22. Tanaka Y. Human mesenchymal stem cells as a tool for joint repair in rheumatoid arthritis. Clin Exp Rheumatol. 2015;33(4 Suppl 92): S58-62.

23. Zhang X, Yamaoka K, Sonomoto K, Kaneko H, Satake M, Yamamoto Y, et al. Local delivery of mesenchymal stem cells with poly-lactic-co-glycolic acid nano-fiber scaffold suppress arthritis in rats. PLoS One. 2014;9(12), e114621. doi:10.1371/journal.pone. 0114621 . This study used a nanofiber scaffold to effectively transplant and retain MSCs to an inflamed joint of arthric rats, where they could effectively suppress inflammation and bone degradation.

24. Steinman L. Multiple sclerosis: a two-stage disease. Nat Immunol. 2001;2(9):762-4. doi:10.1038/ni0901-762.

25. Prineas JW. The neuropathology of multiple sclerosis. In: Koestier JC, editor. Handbook of clinical neurology. Amsterdam: Elsevier; 1985.

26. Furlan R, Cuomo C, Martino G. Animal models of multiple sclerosis. Methods Mol Biol. 2009;549:157-73. doi:10.1007/978-160327-931-4 11.

27. Gerdoni E, Gallo B, Casazza S, Musio S, Bonanni I, Pedemonte E, et al. Mesenchymal stem cells effectively modulate pathogenic immune response in experimental autoimmune encephalomyelitis. Ann Neurol. 2007;61(3):219-27.

28. Uccelli A, Milanese M, Principato MC, Morando S, Bonifacino T, Vergani $\mathrm{L}$, et al. Intravenous mesenchymal stem cells improve survival and motor function in experimental amyotrophic lateral sclerosis. Mol Med. 2012;18:794-804. doi:10.2119/molmed.2011. 00498.

29. Zappia E, Casazza S, Pedemonte E, Benvenuto F, Bonanni I, Gerdoni E, et al. Mesenchymal stem cells ameliorate experimental autoimmune encephalomyelitis inducing T-cell anergy. Blood. 2005;106(5):1755-61. doi:10.1182/blood-2005-04-1496.

30. Tyndall A, Walker UA, Cope A, Dazzi F, De Bari C, Fibbe W, et al. Immunomodulatory properties of mesenchymal stem cells: a review based on an interdisciplinary meeting held at the Kennedy Institute of Rheumatology Division, London, UK, 31 October 2005. Arthritis Res Ther. 2007;9(1):301. doi:10.1186/ar2103.

31. Bai L, Lennon DP, Caplan AI, DeChant A, Hecker J, Kranso J, et al. Hepatocyte growth factor mediates mesenchymal stem cell-induced recovery in multiple sclerosis models. Nat Neurosci. 2012;15(6): 862-70. doi:10.1038/nn.3109. This study showed that MSCinduced recovery in $\mathrm{EAE}$ is mediated by factors like hepatocyte growth factor (HGF) that are secreted from MSCs.

32. Bai L, Lennon DP, Eaton V, Maier K, Caplan AI, Miller SD, et al. Human bone marrow-derived mesenchymal stem cells induce Th2polarized immune response and promote endogenous repair in animal models of multiple sclerosis. Glia. 2009. doi:10.1002/glia. 20841.

33. Bai L, Caplan A, Lennon D, Miller RH. Human mesenchymal stem cells signals regulate neural stem cell fate. Neurochem Res. 2007;32(2):353-62. doi:10.1007/s11064-006-9212-x.

34. Fox JM, Chamberlain G, Ashton BA, Middleton J. Recent advances into the understanding of mesenchymal stem cell trafficking. Br J Haematol. 2007;137(6):491-502. doi:10.1111/j.13652141.2007.06610.x.

35. Jadasz JJ, Kremer D, Göttle P, Tzekova N, Domke J, Rivera FJ, et al. Mesenchymal stem cell conditioning promotes rat oligodendroglial cell maturation. PLoS One. 2013;8(8), e71814. doi:10.1371/ journal.pone.0071814.

36. Lanza C, Morando S, Voci A, Canesi L, Principato MC, Serpero LD, et al. Neuroprotective mesenchymal stem cells are endowed with a potent antioxidant effect in vivo. J Neurochem. 2009;110(5): 1674-84. doi:10.1111/j.1471-4159.2009.06268.x.

37. Benkhoucha M, Santiago-Raber ML, Schneiter G, Chofflon M, Funakoshi H, Nakamura T, et al. Hepatocyte growth factor inhibits CNS autoimmunity by inducing tolerogenic dendritic cells and CD25+Foxp3+ regulatory T cells. Proc Natl Acad Sci U S A. 2010;107(14):6424-9. doi:10.1073/pnas.0912437107.

38. El-Akabawy G, Rashed LA. Beneficial effects of bone marrowderived mesenchymal stem cell transplantation in a non-immune model of demyelination. Ann Anat. 2015;198:11-20. doi:10. 1016/j.aanat.2014.12.002.

39. Connick P, Kolappan M, Crawley C, Webber DJ, Patani R, Michell AW, et al. Autologous mesenchymal stem cells for the treatment of secondary progressive multiple sclerosis: an open-label phase $2 \mathrm{a}$ proof-of-concept study. Lancet Neurol. 2012;11(2):150-6. doi:10. 1016/S1474-4422(11)70305-2.

40. Karussis D, Karageorgiou C, Vaknin-Dembinsky A, GowdaKurkalli B, Gomori JM, Kassis I, et al. Safety and immunological effects of mesenchymal stem cell transplantation in patients with multiple sclerosis and amyotrophic lateral sclerosis. Arch Neurol. 2010;67(10):1187-94. doi:10.1001/archneurol.2010.248.

41. Uccelli A, Laroni A, Freedman MS. Mesenchymal stem cells as treatment for MS — progress to date. Mult Scler. 2013;19(5):515-9. doi: $10.1177 / 1352458512464686$. 